\title{
Bifurcation Analysis of Columns of Composite Materials with Thermal Variation
}

\author{
Alexandre de Macêdo Wahrhaftiga ${ }^{\circledR}$, Kaique Moreira Matos Magalhães ${ }^{b *}$ (1) \\ ${ }^{a}$ Universidade Federal da Bahia, Escola Politécnica, Departamento de Construção e Estruturas, \\ Rua Aristides Novís, Federação, CEP: 40210-630, Salvador, BA, Brasil. \\ ${ }^{b}$ Universidade de São Paulo, Escola Politécnica, Programa de Pós-Graduação em Engenharia Civil, \\ Av. Prof. Almeida Prado, CEP: 05508-900, São Paulo, SP, Brasil.
}

Received: June 03, 2021; Revised: October 20, 2021; Accepted: October 25, 2021

\begin{abstract}
Civil structures are, normally, subjected to gravitational and thermal loads. The association of the effects of these loads should be the object of analysis when the loss of stability of the slender columns was verifying. In the case of reinforced concrete structures, temperature variations induce internal stresses in addition to gravitational ones since they are caused by the difference in the thermal properties of steel and concrete. Since the structure is a slender system, its stiffness was divided into two parts. In the first one, the properties of the concrete were introduced, including cracking and creep, doing the equations obtained at the time-dependent mathematical process. In the second, the geometric, the construction imperfections and the internal efforts mobilized by the temperature variation were considered. Additionally, strains and thermal loads were considered together. At the end, the values of critical buckling loads for different moments of interest were determined.
\end{abstract}

Keywords: thermal variation, critical buckling load, mathematical analysis, slender columns.

\section{Introduction}

Civil structures in reinforced concrete commonly are subjected to a combination of mechanical and thermal loads. These loads must be taken into account jointly in checking the stability of slender columns. ABNT NBR 6118:2014 ${ }^{1}$ recommends that the field temperature variation in reinforced concrete structures, caused globally by the variation in the atmosphere temperature and by direct sunlight, should be considered uniform. It depends on the location of the construction and dimensions of the structural elements that compose it.

Composite structures have a peculiar behavior, justified by their heterogeneity ${ }^{2}$. In this context, reinforced concrete structures, when subjected to any thermal variations, might have their performance altered mainly due to the concrete components ${ }^{3,4}$, which includes the loss of bearing capacity and/or structural performance. Additionally, it is observed that the slender the element, the greater the designer's concern regarding the thermal load on the structure.

For slender reinforced concrete columns, the thermal variation must be added to the creep effects, as well as those produced by the sustained loads, including the structural elements self-weight. The thermal variation can potentiate or even alleviate the conditions of stability or bifurcation of the balance of these systems.

To investigate that hypothesis, a mathematical proposal based on the theory of vibrations was used to determine the critical buckling load of a real slender reinforced concrete structure. The concept behind the used method is one that establishes that the imminence of structural collapse occurs for the nullity of its first natural frequency, occasion in which

*e-mail: kaiquemagalhaes@usp.br it loses stiffness. In the calculation developed to determine the critical buckling load, the longitudinal and transverse strains were considered together and associated with the thermal variation, which was included in the geometric portion of the total stiffness of the structure. The need for this inclusion stems from the existence of internal forces originating from the difference in the thermal expansion coefficients of the concrete and steel. Due to the consideration of transverse deformations, by using the Poisson's ratio, it was necessary to correct the density of the material, because of the change in the volume of the structure. This operation is fundamental to applications of a dynamic nature.

It is important to emphasize that this paper presents an analytical approach to evaluate the frequency and stability by buckling, of slender columns subject to thermal variation and self-weight. The solution developed in the present work is based on the concepts of vibration of structural systems, through modal analysis. By using the path of vibration analysis, the critical buckling load is defined for the frequency nullity, representing the situation in which the structure loses its stiffness. It is noteworthy that this method represents, in the context of the stability of slender columns, an innovative proposal because the internal forces mobilized by the thermal variation are considered directly into the structural stiffness. In reinforced concrete structures, temperature variations induce internal forces because there is different material involved. These internal forces, originated by the different thermal coefficients of the concrete and reinforcement, are treated by mean the geometric stiffness, which is a part of the total stiffness of the column.

It is important to be highlighted that in the present work an analytical formulation is applied to a non-prismatic element 
whose solution is constructed straightly in the continuum dismissing any discretization technique. The previously mentioned aspect is an important point in analysis because when a structural element with geometry varying along the length is presented to study, computational methods based on modeling are required for solving the problem. In these computational methods, normally, the medium needs to be discretized and therefore the results stay conditioned to the density of that discretization.

In the presented analytical approach, important nonlinear aspects were considered at the same time in calculation. These nonlinear aspects are geometric and material. The analysis includes the creep and cracking of the concrete together with a thermal variation. For doing that, a routine of programming needed to be elaborated to solve a multi-step complex mathematical formulation. This is not a trivial task. It is worth mentioning that numerical processing is a timeconsuming process, revealing the computational enormous effort required for making it possible. Since its application can be extended to other structures and similar (or not) loading situations, the presented method can be applied for assessing the stability of columns in a fire condition.

To contextualize the present approach, a real reinforced concrete structure submitted to thermomechanical effects associated with gravitational loads was analyzed ${ }^{5-9}$. A $46 \mathrm{~m}$ high column, having $40 \mathrm{~m}$ of exposure to the environment, $6 \mathrm{~m}$ buried, with a slenderness rate greater than 400, was selected for the study. The analyzes were considered as time functions due to the viscoelastic behavior of the concrete. Constructive imperfections and/or second-order effects were linearized using the geometric stiffness, the material non-linearity of the concrete was considered by reducing the flexural stiffness, and its viscoelastic behavior obeyed the creep criteria provided by the Brazilian normative code ${ }^{1}$. At the end of the process, the values of critical buckling loads at different moments of interest were determined.

\section{Mathematical Proposal for Consideration the Thermal Variation}

Consider the model shown in Figure 1. This model concerns a column clamped at the bottom and free at the top, where $t$ represents time; $\phi(x)$ is a mathematical function that is the form of the first buckling mode; $L$ is the total length (or height) of the structure; $L_{s}$ and $L_{s-1}$ are the positions at the upper and lower limits of a segment $s$, therefore the length of that segment is obtained by the difference between these two positions; and $v(t)$ is a generalized coordinate of the system, located at the free end of the column. Equation 1 refers to a trigonometric function taken as a shape function, which describes the first mode of undamped free vibration, or buckling ${ }^{10}$, being considered valid at any point in the structural domain and that obeys the boundary conditions of the problem, where $x$ originates from the base.

$\phi(x)=1-\cos \left(\frac{\pi x}{2 L}\right)$,

Applying the principle of virtual works and their derivatives ${ }^{11}$, the generalized stiffness and mass of the system can be obtained, among which the geometric stiffness

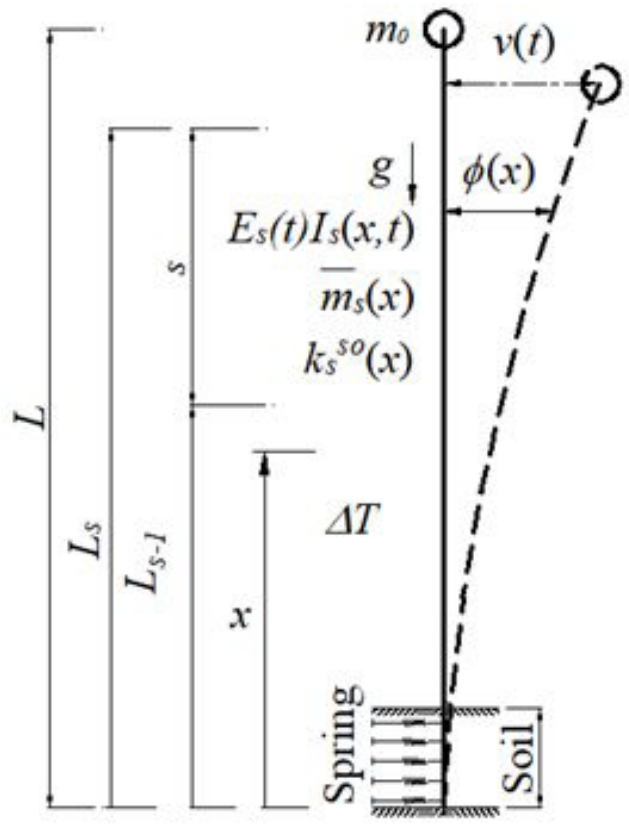

Figure 1. Mathematical model of the structure.

portion $^{12-15}$. This portion is presented as a function of the axial force, including the contribution of the own weight, and where the internal forces induced by a temperature variation are introduced ${ }^{16}$.

The conventional generalized elastic/viscoelastic stiffness portion is defined as:

$K_{0}(t)=\sum_{s=1}^{n} k_{0 s}(t)$, with $k_{0 s}(t)=\int_{L_{s-1}}^{L_{s}} E_{s}(t) I_{S}(x)\left(\frac{d^{2} \phi(x)}{d x^{2}}\right)^{2} d x$,

where for a segment $s$ of the structure, $E_{s}(t)$ is the viscoelastic modulus of the material with respect to time; $I_{s}(x)$ is the variable moment of inertia of the section along the segment in relation to the considered movement, obtained by interpolation of the previous and following sections, already homogenized (if it is constant, it is simply $\left.I_{s}\right) ; k_{0 s}(t)$ is the temporal term for the stiffness; $K_{0}(t)$ is the final conventional stiffness varying over time; and $n$ is the total number of segment intervals given by the structural geometry. In Equation 2, obviously, $t$ vanishes when the analysis considers a material with purely elastic, time-independent behavior.

The geometric stiffness appears as a function of the axial load, including the self-weight contribution, and is expressed as:

$k_{g s}\left(m_{0}\right)=\int_{L_{s-1}}^{L_{s}}\left[N_{0}\left(m_{0}\right)-\sum_{s}^{n} N_{T s}+\sum_{s+1}^{n} N_{s}+\bar{m}_{s}(x)\left(L_{s}-x\right) g\right]\left(\frac{d \phi(x)}{d x}\right)^{2} d x$

where $k_{g s}\left(m_{0}\right)$ is the geometric stiffness in segment $s, K_{g}\left(m_{0}\right)$ is the total geometric stiffness of the structure with $n$ as defined previously, $N_{T s}$, is the internal force mobilized by the thermal variation given by Equation 4 , where $\Delta T$ indicates de thermal variation, the symbols $c$ refers to concrete and st to steel of reinforcement, with $E, A$, and $a$ representing the modulus of elasticity, area, and thermal coefficients of 
the materials. Note that for this first approach these internal forces are not time dependent yet, but they should be.

$$
N_{T s}=\frac{\left(E^{c} A_{s}^{c}\right)\left(E^{s t} A_{s}^{s t}\right) \Delta T\left(\alpha^{s t}-\alpha^{c}\right)}{E_{S}^{c} A_{S}^{c}+E^{s t} A_{S}^{s t}} .
$$

The term $N_{0}\left(m_{0}\right)$ in Equation 3 is the concentrated force at the top, all of which are dependent on the mass $m_{0}$ at the tip, written in the form of following equation:

$N_{0}\left(m_{0}\right)=m_{0} g$.

The total generalized geometric stiffness is calculated by Equation 6, in the form of:

$K_{g}\left(m_{0}\right)=\sum_{s=1}^{n} k_{g s}\left(m_{0}\right)$,

As can be seen, all the previous parameters depend on the mass $m_{0}$ located at the free end of the column. In Equation $3, N_{s}$ represents the normal force in the segment superior to the analyzed segment, which can be obtained from:

$$
N_{S}=\int_{L_{s-1}}^{L_{s}} \bar{m}_{S}(x) g d x .
$$

The generalized mass of the system can be found by:

$$
\begin{aligned}
& M\left(m_{0}\right)=m_{0}+m, \text { with } m=\sum_{s=1}^{n} m_{s}, \text { and } \\
& m_{S}=\int_{L_{s-1}}^{L_{s}} \bar{m}_{S}(x)(\phi(x))^{2} d x,
\end{aligned}
$$

where $\bar{m}_{S}(x)$ is the mass distributed to each segment, defined by Equation 9:

$\bar{m}_{s}(x)=A_{s}(x) \rho_{s}+\bar{m}_{s}^{a}$,

where $A_{s}(x)$ represents the variable area of the cross section, $\rho_{s}$ and $\bar{m}_{s}^{a}$ are the density of the material and the additional distributed mass externally joined for the segment, respectively. If the cross section has a constant area, $A_{\mathrm{s}}(x)$ will simply be $A_{s}$, and, consequently, the mass distribution per unit length will also be constant. Likewise, if the mass $m_{0}$ does not vary, all parameters dependent on it will also be constant. To limit the influence of the soil on the balance of the system, it is necessary to represent it by a series of springs distributed vertically along the foundation. In this way, the contribution of the soil to the stiffness of the structure can be written as:

$K_{S o}=\sum_{s=1}^{n} k_{s}$, with $k_{s}=\int_{L_{s-1}}^{L_{s}} k_{s}^{s o}(x) \phi(x)^{2} d x$ and

$k_{s}^{s o}(x)=S_{S} D_{s}(x)$,

where the parameter $k_{S}^{s o}(x)$ is an elastic characteristic along the foundation depth, which dependents on the geometry of the foundation $D_{s}(x)$, and the soil parameter $S_{s}$. The parameter $S_{s}$ must be provided by a specialist geotechnical engineer. Considering the compressive force as positive, the total structural stiffness is obtained as:

$$
K\left(m_{0}, t\right)=K_{0}(t)-K_{g}\left(m_{0}\right)+K_{S o} .
$$

Therefore, the frequency of the first undamped free vibration mode, in Hertz, can be found using Equation 12: $f\left(m_{0}, t\right)=\frac{1}{2 \pi} \sqrt{\frac{K\left(m_{0}, t\right)}{M\left(m_{0}\right)}}$.

Taking into account the previous postulations and assuming the concentrated mass $m_{0}$ as the variable independent of the problem, once the instant of interest has been defined, the critical buckling load $N_{f t}$ can be determined using the mathematical concept present in Equation 13, which defines the proximity of the loss of balance to when the structure loses its stiffness, making its frequency null:

$$
K\left(m_{0}, t\right)=0 \rightarrow f\left(m_{0}, t\right)=\left.0 \therefore N_{0}\left(m_{0}\right)\right|_{f\left(m_{0}, t\right)=0}=N_{f l} .
$$

The workflow of the mathematical programming routine used to find the buckling load in accordance with the concept of Equation 13 is described in Figure 2.

\section{Results and Discussion}

The analyzed structure was a real, extremely slender, reinforced concrete pole, $46 \mathrm{~m}$ high, including the $40 \mathrm{~m}$ superstructure, exposed to the environment, with a circular hollow section and a $6 \mathrm{~m}$ deep foundation, which is relatively deep, is a belled shaft foundation. The dimensions, heights and the structural arrangement are shown in Figure 3, letters (a) and (b), where $g$ is the acceleration of gravity; $S 1, S 2$, $S 3, S 4$, and $S 5$ are the cross sections from 1 to $5 ; D$ and $t h$ indicate, respectively, the outside diameter and the wall thickness of these sections; $d_{b}$ and $n_{b}$ represent the diameter and number of reinforcement bars; $c^{\prime}$ ' is the cover of concrete in the respective cross sections and "Var" indicates a variable section in the segment. It is assumed that thermal equilibrium occurs for the entire system.

The elasticity modules of the concrete calculated at 28 days after its production, for the superstructure and for the foundation, are, respectively, $34279 \mathrm{MPa}$ and $21287 \mathrm{MPa}$, defined according to ABNT NBR 6118:2014¹', considering the characteristic strengths of the concrete $f_{c k}$ equal to $45 \mathrm{MPa}$ and $20 \mathrm{MPa}$. Equipment is fixed on the upper part of the structure, establishing a concentrated mass, whose limit value in relation to the loss of stability by buckling must be determined. Additional devices are also installed along the

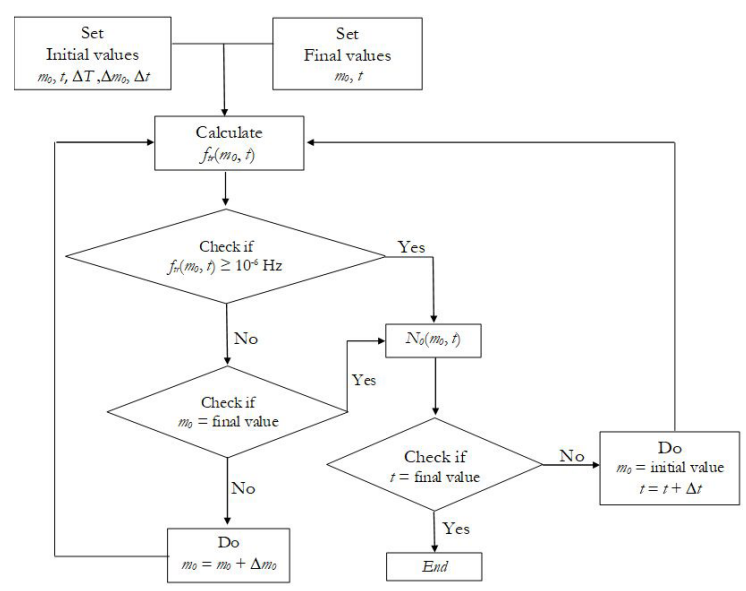

Figure 2. Workflow of the mathematic programming. 

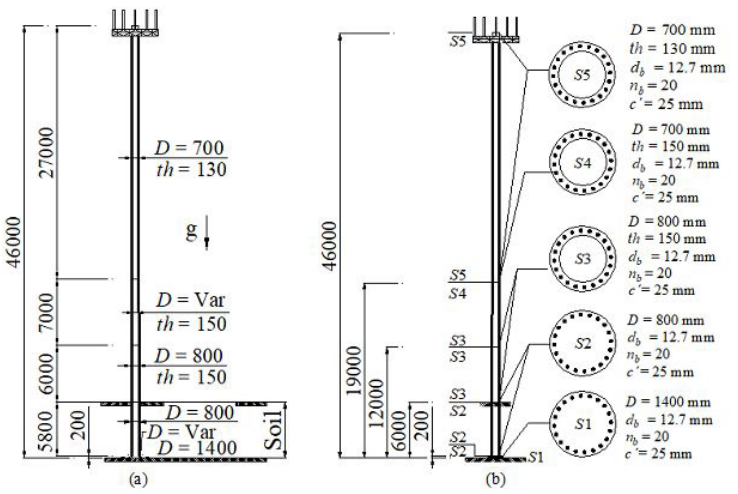

Figure 3. System analyzed (all measures in "mm"): (a) details of geometry; (b) heights and structural arrangement.

superstructure, configuring a distributed mass of $40 \mathrm{~kg} / \mathrm{m}$. The foundation is a belled shaft, with a bell diameter of $1400 \mathrm{~mm}$ and length of $200 \mathrm{~mm}$; the shaft is $800 \mathrm{~mm}$ in diameter and $5800 \mathrm{~mm}$ long. The lateral interaction of the soil with the foundation was represented by an elastic parameter with a property equal to $2669 \mathrm{kN} / \mathrm{m}^{3}$.

The non-linearity of the concrete was calculated by the recommendations of ABNT NBR 6118:2014 ${ }^{1}$ which suggests a $50 \%$ reduction in the moment of inertia of the sections for analysis in similar circumstances, foreseeing the structure will be working in a bent condition. The density of reinforced concrete was defined as $2600 \mathrm{~kg} / \mathrm{m}^{3}$ for the superstructure and $2500 \mathrm{~kg} / \mathrm{m}^{3}$ for the foundation. The homogenization factors were calculated, which multiply the moment of nominal inertia, in order to take into account, the presence of the steel bars of the reinforcement. The creep, due to the rheological behavior of the concrete, was considered in the superstructure ${ }^{1}$.

Thereby, it was possible to evaluate, comparatively, the effects of a positive and negative thermal variation to the load capacity of the structure. These loads were defined for the nullity of the natural frequencies of the system. Figure 4, Figure 5, and Figure 6 represent the structural frequency without (W/O-TV) and with (W/I-TV) thermal variation field. When the thermal variation field $(\Delta \mathrm{T})$ is taken account, a positive and negative interval of $15^{\circ} \mathrm{C}$, respectively, is adopted. In all of those graphs, $N\left(m_{0}\right)$ is the normal force at the top of the column. To obtain those curves, a Poisson's ratio of 0.2 was used for the homogenized concrete section.

Analyzing the results present in Table 1, it is possible to observe that differences between the critical load calculated considering of thermal variation increase up to a maximum of $16.11 \%$, with an average of $14.18 \%$, in relation to that calculated without thermal variation, for the time interval assumed to the simulation.

Table 2 brings the results for the negative thermal variation. There, it is possible to observe there is a peak variation of $8.37 \%$ for the critical load, with average values of $6.29 \%$, which represents an average variation smaller than that presented for a positive thermal variation.

It is important to observe that the mathematical routine give a processing, which used an Intel CPU (R) 2.70 GHz, i7 (7th generation), Core (7 M) 7500U, running Windows

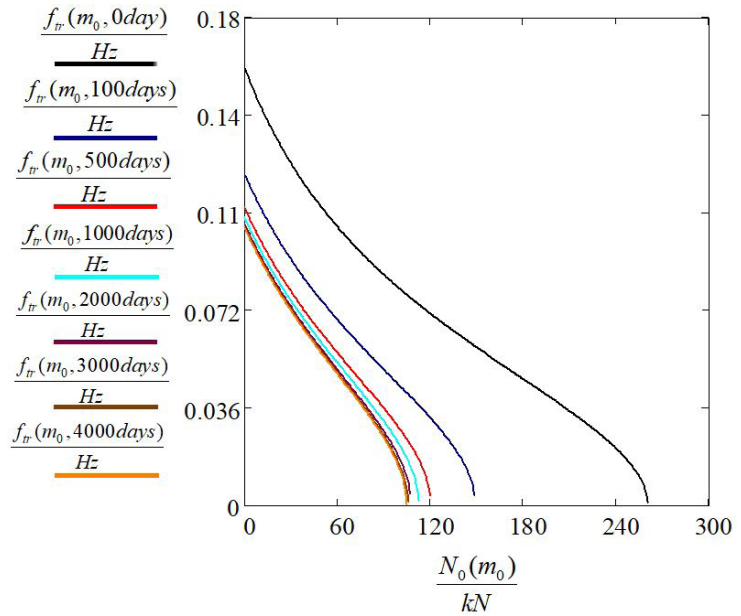

Figure 4. Results for initial condition (environmental temperature).

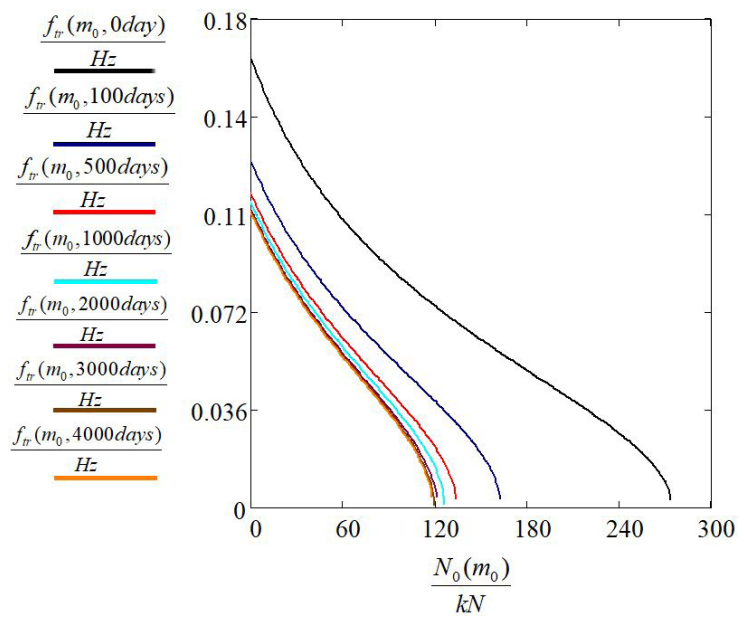

Figure 5. Results for $\Delta T=+15^{\circ} \mathrm{C}$.

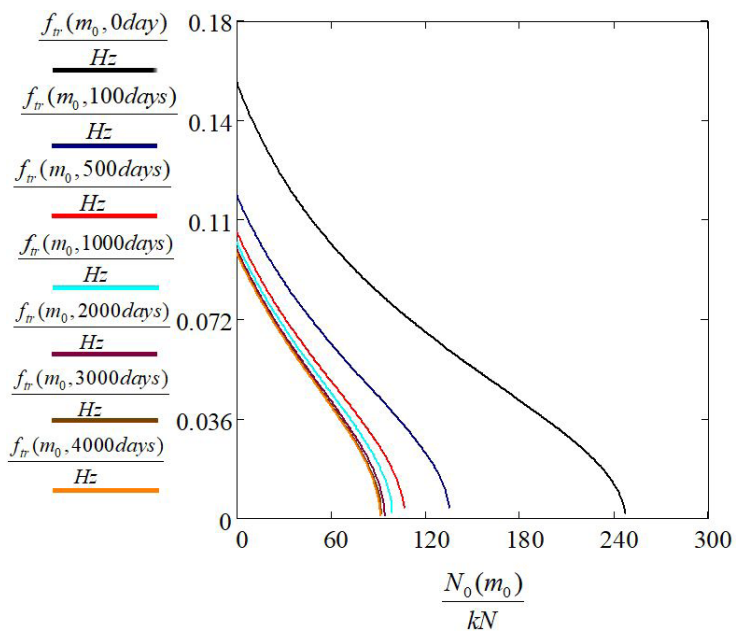

Figure 6. Results for $\Delta T=-15^{\circ} \mathrm{C}$.

10 (64 bits), 8 GB RAM, needed approximately $24 \mathrm{~h}$ to be completed, when using $\Delta m_{0}$ increments of $0.1 \mathrm{~kg}$ for the mass, and $\Delta t$ of 100 days for time, adopting initial values 
Table 1. Obtained results from $\Delta T=0^{\circ} \mathrm{C}$ and $\Delta T=+15^{\circ} \mathrm{C}$

\begin{tabular}{cccc}
\hline \multirow{2}{*}{ Time (day) } & \multicolumn{2}{c}{ Critical buckling load $(\mathrm{kN})$} & \multicolumn{2}{c}{ Difference $(\mathrm{W} / \mathrm{O}-\mathrm{TV}$ to W/IP-TV) } \\
\cline { 2 - 4 } \cline { 3 - 4 } & W/O-TV & W/IP-TV & $(\%)$ \\
\hline 0 & 249.58 & 274.59 & 9.11 \\
\hline 100 & 142.69 & 163.28 & 12.61 \\
\hline 500 & 114.74 & 134.35 & 14.60 \\
\hline 1000 & 107.38 & 126.51 & 15.12 \\
\hline 2000 & 102.48 & 121.60 & 15.73 \\
\hline 3000 & 100.52 & 119.64 & 16.11 \\
\hline 4000 & 99.54 & 118.66 & Average $=14.18$ \\
\hline
\end{tabular}

$\mathrm{W} / \mathrm{O}-\mathrm{TV}=$ without thermal variation; $\mathrm{W} / \mathrm{IP}-\mathrm{TV}=$ with positive thermal variation.

Table 2. Obtained results from $\Delta T=0^{\circ} \mathrm{C}$ and $\Delta T=-15^{\circ} \mathrm{C}$.

\begin{tabular}{|c|c|c|c|}
\hline \multirow{2}{*}{ Time (day) } & \multicolumn{2}{|c|}{ Critical buckling load (kN) } & \multirow{2}{*}{$\begin{array}{c}\text { Difference (W/O-TV to W/IN-TV) } \\
(\%)\end{array}$} \\
\hline & W/O-TV & $\mathrm{W} / \mathrm{IN}-\mathrm{TV}$ & \\
\hline 0 & 249.58 & 247.62 & 0.79 \\
\hline 100 & 142.69 & 135.82 & 4.81 \\
\hline 500 & 114.74 & 106.89 & 6.86 \\
\hline 1000 & 107.38 & 99.54 & 7.30 \\
\hline 2000 & 102.48 & 94.14 & 8.14 \\
\hline 3000 & 100.52 & 92.67 & 7.81 \\
\hline 4000 & 99.54 & 91.20 & 8.37 \\
\hline
\end{tabular}

$\mathrm{W} / \mathrm{O}-\mathrm{TV}=$ without thermal variation; $\mathrm{W} / \mathrm{IN}-\mathrm{TV}=$ with negative thermal variation.

equals to $5,000 \mathrm{~kg}$ for $m_{0}$ and 0 for $t$, with a final time equal to 4000 days.

\section{Conclusions}

In this paper, an innovative analytical mathematical approach is used to determine the critical buckling load of a real slender reinforced concrete structure subjected to thermal variation and self-weight. For this proposal, it was applied a development based on the concepts of the mechanics of vibrations, by modal analysis.

The necessity and the main importance of the present analysis comes up due the fact that reinforced concrete structural elements are submitted to additional forces when subjected to thermal variation because their cross-sections are formed by materials with different expansion coefficients. This difference alters the internal equilibrium of forces and conditions of compatibility of the displacements, in the referred sections, producing changes in the geometric stiffness of the structure and, consequently, in the vertical loading capacity of the system.

From the analyzes performed, it was possible to verify that a positive thermal variation of $15^{\circ} \mathrm{C}$, taken into account in association with the temporal change in the concrete elasticity and with the effects of gravitational forces, elevated the values of the critical buckling load by $14 \%$, which represents an increase in the vertical load-carrying capacity. When considering a negative thermal variation of same magnitude, the structure presented a loading capacity $8.37 \%$ lower to that without thermal variation, representing to be that the most critical situation among those analyzed.
The computational cost and the results generated from the mathematical proposal developed to solve the analyzed problem are conditioned to the discretization used in the programming routine. This means that the smaller this is, the better the results will be. However, the computational processing time increases proportionally.

For future studies, analysis considering the temporal variation of the Young's Modulus of concrete into the internal forces mobilize by thermal variation together with changes to the structural volume due to the transversal deformation is desired. On the other hand, evaluation of structures in fire condition is equally waited.

\section{References}

1. Associação Brasileira de Normas Técnicas - ABNT. ABNT NBR 6118: projeto de estruturas de concreto: procedimento. Rio de Janeiro: ABNT; 2014. 238 p.

2. Pitangueira RL, Silva RR. Numerical characterization of concrete heterogeneity. Mater Res. 2002;5:309-14.

3. Tolentino E, Lameiras FS, Gomes AM, Silva CAR, Vasconcelos WL. Structural evaluation and performance of portland cement concretes after exposure to high temperatures. Mater Res. 2002;5:27-36.

4. Tolentino E, Lameiras FS, Gomes AM, Silva CAR, Vasconcelos WL. Effects of high temperature on the residual performance of portland cement concretes. Mater Res. 2002;5:301-7.

5. Usmani AS, Rotter JM, Lamont S, Sanad AM, Gillie M. Fundamental principles of structural behaviour under thermal effects. Fire Saf J. 2001;36:721-44.

6. Richeton J, Ahzi S, Vecchio KS, Jiang FC, Adharapurapu RR. Influence of temperature and strain rate on the mechanical behavior of three amorphous polymers: characterization and modeling of the compressive yield stress. Int J Solids Struct. 2006;43:2318-35. 
7. Pires D, Barros RC, Lemes IJM, Rocha PAS, Silveira RA. Advanced numerical analysis of steel, concrete and composite structures under fire conditions. In: Eurosteel; 2017; Copenhagen. Proceedings. Lyngby: DTU Civil Engineering; 2017.

8. Machado LR, Dutra VFP, Maghous S. A limit analysis approach to the stability assessment of reinforced concrete panels in fire condition. Lat Am J Solids Struct. 2020;17:1-27.

9. Abid SR. Temperature variation in steel beams subjected to thermal loads. Steel Compos Struct. 2020;34:819-35.

10. Wahrhaftig AM. Analysis of the first modal shape using two case studies. Int J Comput Methods. 2019;16(6):1840019.

11. Clough RW, Penzien J. Dynamics of structures. Berkeley: Computers \& Structures, Inc.; 1995.

12. Wahrhaftig AM. Brasil RMLRF. Vibration analysis of mobile phone mast system by Rayleigh method. Appl Math Model. 2016;42:330-45.
13. Wahrhaftig AM, Brasil RMLRF. Initial undamped resonant frequency of slender structures considering nonlinear geometric effects: the case of a 60.8 m-high mobile phone mast. J Braz Soc Mech Sci Eng. 2017;39:725-35.

14. Wahrhaftig AM, Brasil RMLRF, Balthazar JM. The first frequency of cantilever bars with geometric effect: a mathematical and experimental evaluation. J Braz Soc Mech Sci Eng. 2013;35:45767.

15. Wahrhaftig AM, Silva MA, Brasil RMLRF. Analytical determination of the vibration frequencies and buckling loads of slender reinforced concrete towers. Lat Am J Solids Struct. 2019;16:1-31.

16. Wahrhaftig AM, Magalhães KMM, Brasil RMLRF, Murawski K. Evaluation of mathematical solutions for the determination of buckling of columns under self-weight. J Vib Eng Technol. 2021;9:733-49. 